\title{
Transition to Mach reflexion of shock waves in steady and pseudosteady flow with and without relaxation
}

\author{
By H. G. HORNUNG, H. OERTEL† AND R. J. SANDEMAN \\ Department of Physics, Australian National University, Canberra, Australia
}

(Received 8 May 1978)

Experiments were conducted in the free-piston shock tube and shock tunnel with dissociating nitrogen and carbon dioxide, ionizing argon and frozen argon to measure the transition condition in pseudosteady and steady flow. The transition condition in the steady flow, in which the wall was eliminated by symmetry, agrees with the calculated von Neumann condition. In the real gases this calculation assumed thermodynamic equilibrium after the reflected shock. In the pseudosteady flow of reflexion from a wedge the measured transition angle lies on the Mach-reflexion side of the calculated detachment condition by an amount which may be explained in terms of the displacement effect of the boundary layer on the wedge surface. A single criterion based on the availability of a length seale at the reflexion point explains the difference between the pseudosteady and steady flow transition condition and predicts a hysteresis effect in the transition angle when the shock angle is varied during steady flow. No significant effects on the transition condition due to finite relaxation length could be detected. However, new experiments in which interesting relaxation effects should be evident are suggested.

\section{Introduction}

Since Ernst Mach (1878) presented his work on the reflexion of shock waves 100 years ago, a great deal of experimental and theoretical work has been aimed at understanding the mechanism of the transition from the regular to the forked or 'Mach' reflexion. To justify our presentation of yet another paper on this subject it is appropriate to give a brief explanation.

At the outset this work was motivated by the desire to understand how the transition from regular to Mach reflexion is affected by relaxation after the translational shock wave. In the course of the experimental investigation, which tackled the problem in the steady as well as the pseudosteady case, both at high free-stream Mach number, we became aware of the following important fact. In the steady case the transition to Mach reflexion occurs at the von Neumann condition (the smallest angle $\alpha$ between incident shock and wall at which Mach reflexion is theoretically possible), while in the pseudosteady case the transition occurs at the point where the flow after the reflected shock is sonic relative to the reflexion point. At high Mach number the two conditions are separated by approximately $10^{\circ}$ in $\alpha$.

This clear experimental result improves the understanding of the mechanism of

$\dagger$ Permanent address: Institut für Strömungslehre und Strömungsmaschinen, Universität Karlsruhe, Federal Republic of Germany. 


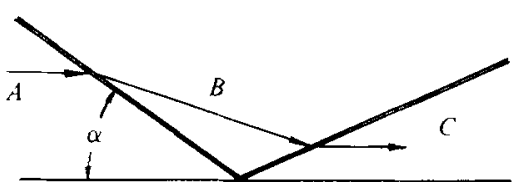

(a)

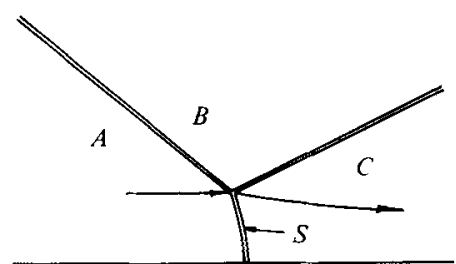

(b)

FIGURE 1. (a) Regular- and (b) Mach-reflexion configurations. The labelled regions are correspondingly labelled in the pressure-deflexion maps of figure 2 .

transition considerably. It leads to a simple physical explanation of the effect in the case of high Mach number perfect-gas flows.

\subsection{The phenomenon}

Figure 1 shows the two reflexion configurations. When $\alpha$ is sufficiently small, the streamline deflexion caused by the incident shock can be cancelled by the reflected shock, giving the regular-reflexion configuration of figure $1(a)$ with supersonic flow throughout. As $\alpha$ is increased beyond a eritical value, the configuration changes suddenly to that shown in figure $1(b)$, in which a nearly normal, third shock appears at the wall, joining the incident and reflected shocks in a triple point off the wall. This allows the net deflexion of the streamline through the triple point to be a little towards the wall.

The smallest value of $\alpha$ at which the configuration of figure $1(b)$ is theoretically possible was obtained by von Neumann (1943) and will be denoted $\alpha_{N}$. The largest value of $\alpha$ at which the configuration of figure $1(a)$ is possible is $\alpha_{d}$, for which the streamline deflexion by the incident shock just equals the maximum deflexion achievable by the reflected shock; $\alpha_{d}>\alpha_{N}$ (see, for example, Courant \& Friedrichs 1948, chap. IV, figure 66). Because this corresponds to shock detachment in flow over a wedge, it is sometimes called the detachment condition. There is a large body of experimental evidence supporting the detachment condition as the criterion for transition, and this result has found its way into the well-known textbooks on gasdynamics (e.g. Liepmann \& Roshko 1957; Landau \& Lifshitz 1959). Most of this evidence, expecially the high $M_{\infty}$ part, was obtained in the pseudosteady situation of a moving plane shock incident on a wedge. This flow is called pseudosteady because it maintains a constant shock configuration but grows linearly with the time from the instant when the shock strikes the leading edge of the wedge. Viewed from the frame of reference of the reflexion point, the flow in the immediate vicinity of a regular reflexion point is steady, with $M_{\infty}=M_{s} / \sin \alpha$, where $M_{s}$ is the incident shock Mach number. Some more recent experiments (Henderson \& Lozzi 1975; Hornung \& Kychakoff, 1977) in the steady case clearly support the von Neumann condition as the correct criterion.

To illustrate the difference between the two criteria it is instructive to map the configurations of figure 1 into the pressure, deflexion $(p-\delta)$ plane. The locus of pressures achievable from a free-stream state $A$ via an oblique shock deflecting the flow through an angle $\delta$ is given by the curve $A B C$ in figure $2(a)$. This is called the shock locus. Thus the region $B$ in figure $1(a)$ maps into the point $B$ in figure $2(a)$. From this point a second shock locus may be drawn to represent the possible conditions 


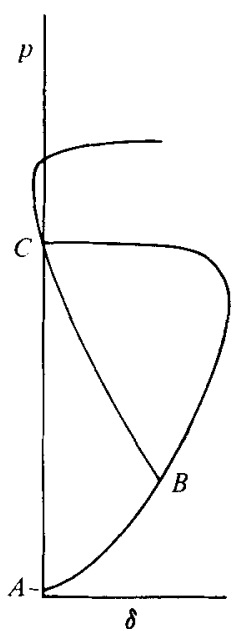

(a) $\alpha=\alpha_{N}$

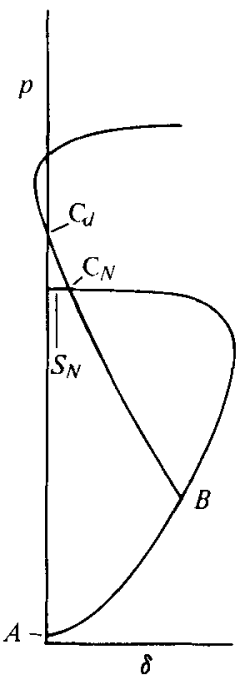

(b) $\alpha_{N}<\alpha<\alpha_{s}$

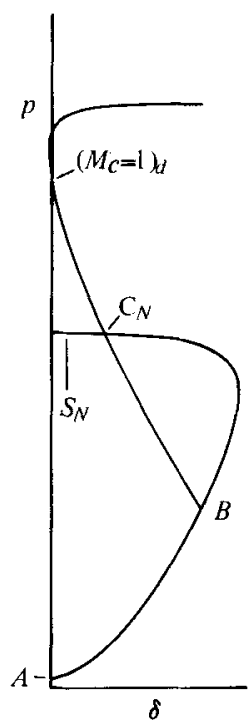

(c) $\alpha=\alpha_{5}$

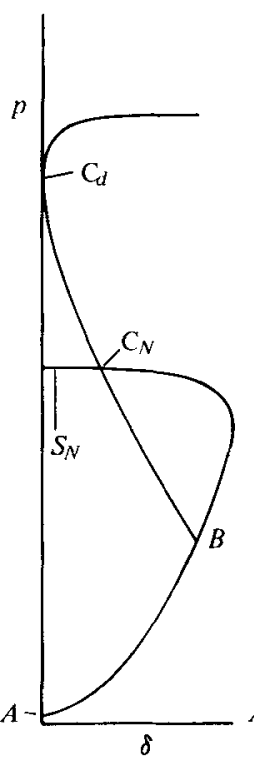

(d) $\alpha=\alpha_{d}$

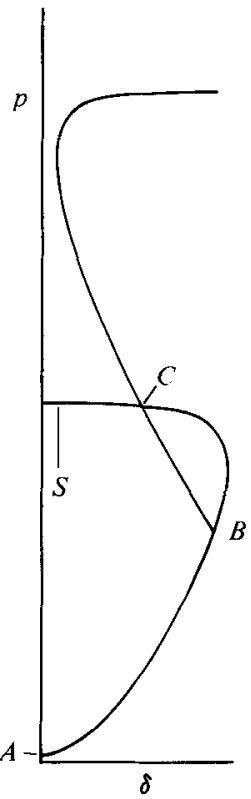

(e) $\alpha>\alpha_{d}$

Figure 2. Pressure-deflexion maps of shock reflexion for various values of the angle $\alpha$. The labels correspond to similar labels in figure 1. The subscripts $N$ and $d$ refer to configurations according to the von Neumann and detachment criteria respectively.

achievable via the reflected shock. Figure $2(a)$ shows the case $\alpha=\alpha_{N}$, where the point $C$, representing region $C$ in figure $1(a)$, just coincides with the pressure achieved from $A$ via a normal shock. Compare with this the detachment condition $\alpha=\alpha_{d}$ shown in figure $2(d)$. According to the detachment criterion, regular reflexion still occurs and region $C$ maps into the point $C_{d}$. According to the von Neumann criterion, transition has occurred at a smaller angle, so that the Mach-reflexion configuration of figure $1(b)$ applies, and region $C$ maps into the point $C_{N}$, the Mach stem $S$ being represented by the piece of shock locus between $C_{N}$ and the $p$ axis, marked $S_{N}$. Yet another important condition is the sonic condition $\alpha=\alpha_{s}$, shown in figure $2(c)$, at which the Mach number in region $C$ is 1 . The angles $\alpha_{s}$ and $\alpha_{d}$ are so close to each other for all conditions of present interest as to be experimentally unresolvable. Note that the highest pressure achievable according to the von Neumann criterion is the normal shock value, while the detachment criterion allows it to become much higher, before dropping discontinuously at transition. Figures $2(b)$ and (e) show the cases $\alpha_{N}<\alpha<\alpha_{d}$ and $\alpha>\alpha_{d}$ respectively and are self-explanatory.

The extent of the difference between $\alpha_{d}$ and $\alpha_{N}$ varies with $M_{\infty}$ as is shown in figure 3 for ratios of specific heats $\gamma=\frac{7}{5}$ and $\frac{5}{3}$. Also shown in the figure are some selected experimental results. Note that the pseudosteady results support the detachment criterion, while the steady results support the von Neumann criterion.

It can be seen from figure 1 that the transition to Mach reflexion is marked by the sudden appearance of a length scale. In the absence of length scales governed by gas properties such as viscosity, heat conduction or relaxation, the only length entering the problem is that pertaining to the geometry of the boundary conditions (see also 


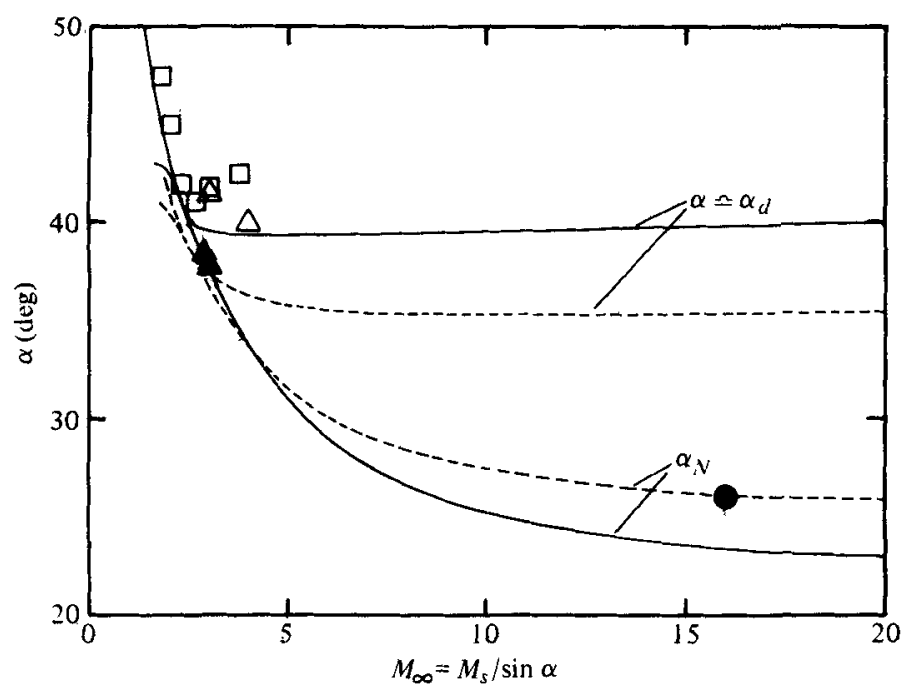

Figure 3. The transition criteria as functions of Mach number for perfect gases:,$- \gamma=\frac{7}{4}$; ,$-- \gamma=\frac{k}{3}$. Experimental results: $\square$, Bleakney \& Taub (1949), air; $\Delta, \Delta$, Henderson \& Lozzi (1975), air; $O$, Hornung \& Kychakoff (1977), argon. Open symbols, pseudosteady experiments; filled symbols, steady experiments.

Liepmann \& Roshko 1957, p. 107). Hence the length of the Mach stem $S$ in figure $1(b)$, its curvature and the curvature of the streamline through the triple point must be governed by the geometry of the boundary conditions. Since the configuration is endowed with a length scale only above and not below a critical value of $\alpha$, this value of $\alpha$ must mark a change in conditions which enables the boundary conditions to communicate themselves to the reflexion point. This suggests strongly that the required change in conditions is a change from supersonic to subsonic flow in some important region.

\subsection{The influence of relaxation}

Relaxation effects are likely to be interesting in the problem of transition to Mach reflexion because they introduce a length scale peculiar to the gas and free-stream conditions which is virtually independent of whether regular or Mach reflexion occurs. This relaxation length is therefore able to influence the flow in the immediate vicinity of the reflexion point for all values of $\alpha$. If the relaxation length, i.e. the distance behind the shock at which the gas has reached equilibrium again, is small compared with the observer's smallest resolvable length, the flow may be considered to be in equilibrium everywhere except in thin shock waves. If the relaxation length is large compared with the region of interest, the gas composition is effectively constant and the flow is 'frozen'. It is clear that the shock locus in the $p, \delta$ plane and consequently the values of $\alpha_{d}$ and $\alpha_{N}$ will be different for equilibrium and frozen flow. For endothermic relaxation, the equilibrium values of both $\alpha_{N}$ and $\alpha_{d}$ are larger than their frozen values:

$$
\alpha_{d, \mathrm{eq}}>\alpha_{d, \mathrm{Ir}}, \quad \alpha_{N, \mathrm{eq}}>\alpha_{N, \mathrm{fr}} \text {. }
$$

Two possibilities exist for the order of these four angles:

$$
\begin{aligned}
& \alpha_{d, \mathrm{eq}}>\alpha_{d, \mathrm{fr}}>\alpha_{N, \mathrm{eq}}>\alpha_{N, \mathrm{fr}}, \\
& \alpha_{d, \mathrm{eq}}>\alpha_{N, \mathrm{eq}}>\alpha_{d, \mathrm{fr}}>\alpha_{N, \mathrm{fr}},
\end{aligned}
$$


depending on $M_{\infty}$ and on the amount of energy needed to dissociate the gas compared with the ordered kinetic energy of the flow. In all the experimental situations studied below (1) applies.

Although a number of investigators have studied real-gas effects on transition to Mach reflexion (Law \& Glass 1971; Gvozdeva et al. 1969; Ben-Dor \& Glass 1977, private communication), the main emphasis in this work was the equilibrium situation, in which no new length scale is introduced. An argument to predict the behaviour of the steady flow transition in the presence of a finite relaxation length was presented by Hornung \& Kychakoff (1977) from which it was concluded that the Mach-stem length is proportional to the relaxation length in a range of $\alpha$ between the frozen and equilibrium transition angle. On this basis a simple model was constructed by which the transition angle (which was taken to be $\alpha_{N}$ ) varies smoothly from its frozen to its equilibrium value depending on the minimum length resolvable by the observer. Hornung \& Kychakoff's (1977) experimental results do not agree with this model,

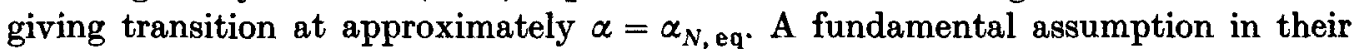
argument is that there exists a single transition condition for all situations. This means, for example, that if a steady flow for which $\alpha$ lies between the equilibrium and frozen value of the transition angle is viewed by an observer studying the reflexion point from very close, so that he can resolve very small distances, he will see a Mach reflexion with a stem length proportional to the relaxation length. If this occurs, the relaxation length communicates itself to the reflexion point in different manners above and below the frozen transition angle. Such a change would be indicated, for example, at the frozen sonic condition but not at the frozen von Neumann condition, so that the results for the argument are likely to depend on whether the inequalities (1) or (2) apply.

The most appealing reason for transition to Mach reflexion to occur remains the ability of the flow to communicate a length scale to the reflexion point in a manner previously not available to it. Relaxation by itself may not perform this function, but it is likely to have an effect when the relaxation length is comparable to the length scale of the boundary conditions.

In the following we present experimental results and comparisons with calculations on the transition to Mach reflexion in dissociating and perfect gases both in the steady and the pseudosteady case, as well as a discussion giving a critical evaluation of the results in the light of the ideas raised above.

\section{The pseudosteady case}

\subsection{Choice of experimental conditions}

The conditions for the pseudosteady experiments were chosen to give an easily resolvable relaxation length after the incident shock wave at a density which makes flow visualization by interferometry easily possible. With dissociative relaxation the extreme temperature sensitivity of the relaxation rate causes the relaxation length after the reflected shock to be very much shorter than that after the incident shock, so that, under the chosen circumstances, the relaxation after the reflected shock is not resolvable. The reason for this choice was that the other situation achievable with dissociative relaxation (that of frozen flow after the incident shock with a resolvable 

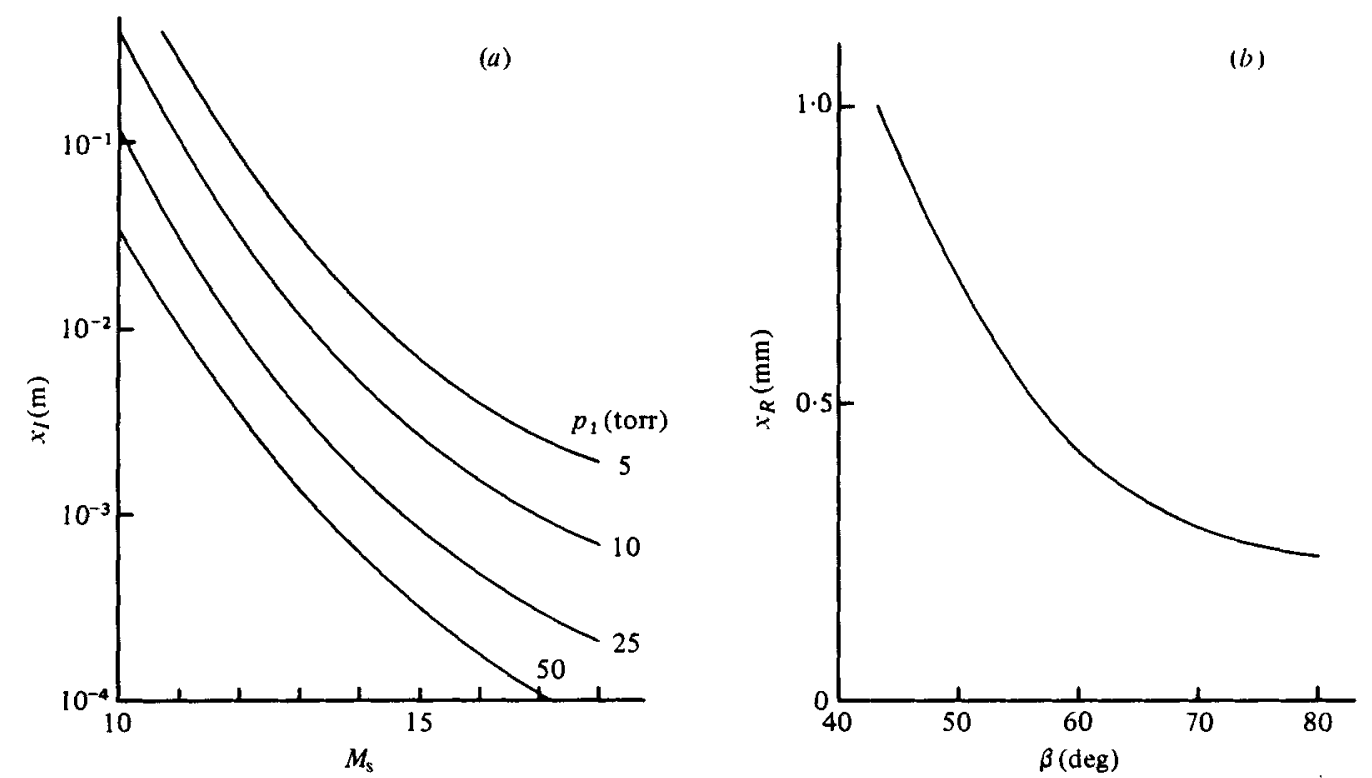

Figure 4. The calculated $90 \%$ relaxation length after the inoident $\left(x_{I}\right)$ and reflected $\left(x_{R}\right)$ shock for nitrogen. $x_{I}$ is shown as a function of the incident shock Mach number and initial shock-tube pressure. For $p_{1}=5$ torr and $M_{s}=12 \cdot 9,(b)$ shows $x_{R}$ as a function of the wedge angle $\beta=\frac{1}{2} \pi-\alpha$. The caloulations use the rate coefficients given by Kewley \& Hornung (1974a) and assume molecular vibration to be in equilibrium.

relaxation zone after the reflected shock) had already been investigated by Oertel (1976) with oxygen as the test gas in the pseudosteady case, supporting the equilibrium sonic criterion for transition, and with nitrogen and carbon dioxide in the steady case by Hornung \& Kychakoff (1977), supporting the equilibrium von Neumann criterion. It was, in fact, partly this apparent disagreement which brought the present authors together in an attempt to resolve the cause of the disagreement by performing the two kinds of experiments in the same laboratory and in the presence of protagonists of both criteria.

Figure $4(a)$ shows the relaxation length $x_{I}$ after the incident shock plotted against shock Mach number for various initial shock-tube pressures $p_{1}$ as calculated for nitrogen according to the reaction rates given by Kewley \& Hornung (1974a). The relaxation length is defined as the distance from the shock to the point where the change in dissociation fraction reaches $90 \%$ of the change to equilibrium. It can be seen that, to achieve a value of $x_{1}=3 \mathrm{~cm}, M_{s}$ has to be in the range $10<M_{s}<13$ for

$$
50 \text { torr }>p_{1}>5 \text { torr. }
$$

To illustrate the comparative smallness of the reflected shock relaxation length $x_{R}$, figure $4(b)$ shows a plot of this quantity vs. the wedge angle $\beta=\frac{1}{2} \pi-\alpha$ (which affects the reflected shock angle and therefore the relaxation rate after it) for regular reflexion in the particular case $p_{1}=5$ torr, $M_{s}=12.9$, where $x_{I}=3.4 \mathrm{~cm}$. Note that $x_{R}$ is less than $1 \mathrm{~mm}$ throughout the range of $\beta$ where regular reflexion is expected. It should be pointed out that the behaviour of dissociative relaxation is highly nonlinear, so that, if the relaxation length were redefined at $50 \%$ rather than at $90 \%$ relaxation, $x_{I}$ would only be $0.7 \mathrm{~cm}$ compared with $3.4 \mathrm{~cm}$ for $x_{I}(90 \%)$ in the case $p_{1}=5$ torr, and $M_{s}=12.9$. 

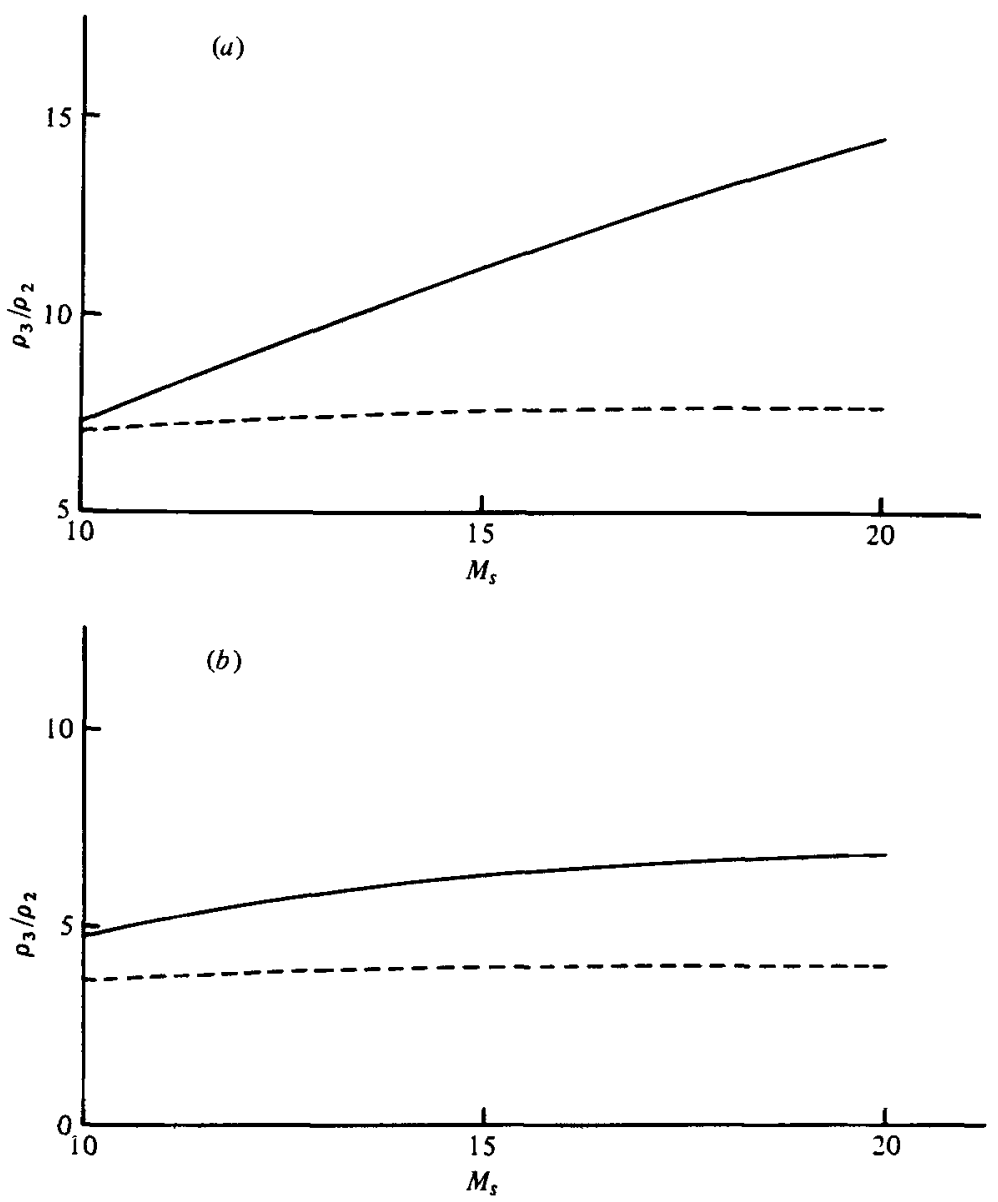

Figure 5. Calculated density ratio across $(a)$ the incident shock $\left(\rho_{2} / \rho_{1} ; p_{1}=5\right.$ torr $)$ and $(b)$ the reflected shock $\left(\rho_{3} / \rho_{2} ; \beta=60^{\circ}\right)$ as a function of the incident shock Mach number $M_{s}$ in nitrogen. ---, Frozen dissociation; _- equilibrium dissociation. Molecular vibration in equilibrium.

It is important to operate at a condition at which a significant amount of dissociation does occur. Figure $5(a)$ shows the increase in density due to dissociation after the incident shock as a function of $M_{s}$. Clearly, $M_{s}$ must be well above 10 to get an easily measurable effect. However, the rapid fall of $x_{I}$ with increasing $M_{s}$ at the smallest desirable value of $p_{1}$ needed for a reasonable fringe shift (see figure $4 a$ ) forces a compromise. A value of $M_{s}=13$ at $p_{1}=5$ torr gives $x_{I}=3.4 \mathrm{~cm}$ and a density increase above the frozen value of $23 \%$. The corresponding density ratio across a regularly reflected shock is shown in figure $5(b)$ as a function of $M_{s}$ for $\beta=60^{\circ}$. Because of the short relaxation length in this region the equilibrium curve may be used for calculations of the reflected shock.

The dissociation after the shocks modifies the transition criteria in a manner which may be calculated numerically. Figure 6 shows the results of such calculations for the sonic criterion as a function of $M_{s}$ for nitrogen at $p_{1}=5$ torr and $T_{1}=293^{\circ} \mathrm{K}$. It is assumed in these calculations that molecular vibration is in equilibrium. The figure shows a number of cases to demonstrate the effect of dissociation both after the incident and after the reflected shock separately as well as the effect of molecular vibration. 


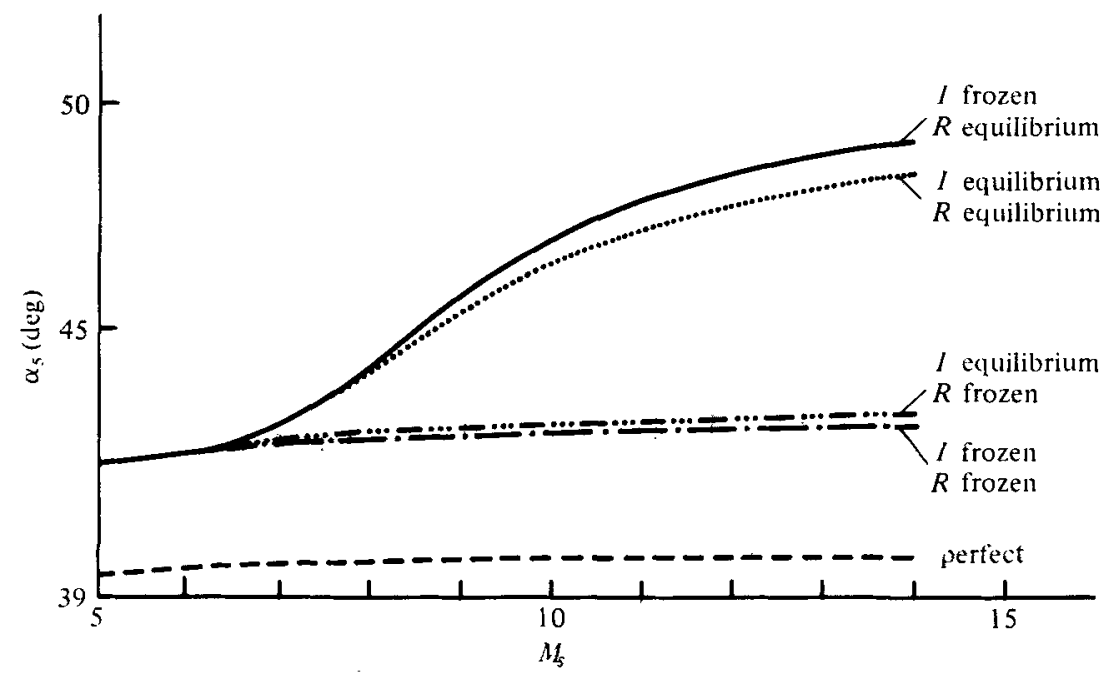

FIGURE 6. The calculated sonic criterion for nitrogen. The various curves show results for different conditions after the incident $(I)$ and reflected $(R)$ shock. Equilibrium molecular vibration is assumed in all curves exoept that for a perfect gas $\left(\gamma=\frac{7}{b}\right) \cdot p_{1}=5$ torr.

The latter can be seen to increase $\alpha_{s}$ above the perfect-gas value by an approximately constant amount of $2 \cdot 7^{\circ}$. The effect of dissociation after the reflected shock is seen to increase rapidly above $M_{s} \simeq 7$, amounting to approximately $6^{\circ}$ at $M_{s}=\mathbf{1 2 . 9}$. Dissociation after the incident shock alters $\alpha_{s}$ only slightly, however. To show this the unlikely case of an equilibrium incident and a frozen reflected shock is also displayed in figure 6. Since dissociation after the reflected shock is the most important effect, the departure point from the frozen curve lies at $M_{s} \simeq 7$, i.e. well below the point where significant dissociation would occur after the incident shock. The calculated values of $\alpha_{d}$ differ from $\alpha_{s}$ by approximately $0 \cdot 1^{\circ}$, so that it would not be possible to distinguish between them in the figure.

At $M_{s}=12.9$ the highest value of $\alpha_{s}$, which is obtained with a frozen incident and an equilibrium reflected shock, is $48.9^{\circ}$, compared with $42.8^{\circ}$ for molecular vibration only and $39.9^{\circ}$ for a perfect gas. At the chosen conditions for the experiment dissociation and vibration amount to a change of $9^{\circ}$, which represents an easily measurable effect.

\subsection{Facility and instrumentation}

The facility used for both the pseudosteady and the steady experiments was the large free-piston-driven shock tunnel known as T3. For the pseudosteady experiments it was arranged as a shock tube opening into a large test section with remote side walls. The shock-tube diameter is $7.6 \mathrm{~cm}$, and a wedge of $4 \mathrm{~cm}$ width was mounted with its leading edge just behind the exit plane of the shock tube. The details of the free-piston driver technique may be found elsewhere (Stalker 1967). For the experiments described here the facility had to be operated at low performance levels to produce the required shock waves with $M_{s} \simeq 13$ travelling into nitrogen at a pressure of 5 torr. The shock speed was measured by four thermocouple timing transducers. Throughout the experiments the shock attenuation did not exceed $2 \%$ over the last $2 \mathrm{~m}$ of the shock-tube length and at some conditions was less than $0.5 \%$. The shock tube and test section were filled 
to the required pressure with commercially pure nitrogen or argon, the pressure being measured with a McLeod gauge. With care this enabled the operator to reproduce the shock speed to an accuracy of $\pm 1 \%$.

The flow was studied with a Mach-Zehnder interferometer. A dye laser constructed in the Physics Department served as a light source. The dye cell of this laser is ' pumped' by a pulsed nitrogen laser which in turn is pumped by a tailored electrical discharge to produce a very short flash in the near ultraviolet range. The dye laser served to translate this flash into the visible range, giving a very high intensity flash of $5 \mathrm{~ns}$ duration at $585 \pm 3 \mathrm{~nm}$. During the exposure time of the flash, in a typical experiment, the incident shock wave traverses a distance of only $2 \times 10^{-3} \mathrm{~cm}$. The laser was timed to catch the shock at the appropriate position on the wedge face by a variable delay, triggered from the last shock timing station $70 \mathrm{~cm}$ upstream of the shock-tube exit plane. Care was taken to align the light beam and the model with the normal to the shock-tube axis, in order to make sure that no Mach reflexions were hidden because of misalignment. However, this was not always easy, as the typical shock loads of $\sim 4 \times 10^{4} \mathrm{~N}$ exerted by the flow on the model necessitated a very heavy and therefore cumbersome construction of the model support.

\subsection{Experimental results}

Three examples of interferograms taken of pseudosteady shock reflexion in nitrogen are shown in figure 7 (plate 1). The shock wave enters the field of view from the right, encounters the wedge and is reflected off it. Figure $7(a)$ shows an example of regular reflexion, the reflected shock intersecting the wall at the same point as the incident shock. Note that the relaxation between the shocks does not cause any noticeable concave curvature in the reflected shock. This is not surprising, however, as the predicted curvature amounts to a change of only approximately $1^{\circ}$ over the relaxation length. The reflected shock angles are consistently higher than the calculated equilibrium values, though the excess is barely significant. Figure $7(b)$ shows an example of incipient Mach reflexion partially hidden by bad misalignment of the wedge (note the broad image of the leading edge). This figure is interesting because the lower face of the wedge is at $5^{\circ}$ incidence to the flow, giving $\alpha=85^{\circ}$ and a bare hint of a Mach reflexion. On the top surface $\left(\alpha=55^{\circ}\right)$ the wedge bow shock (i.e. the reflected shock) is strongly curved near the reflexion point. Figure $7(c)$ shows a larger Mach reflexion, the picture being taken when the shock has proceeded further up the wedge, and at an angle further from the critical angle for transition. The configuration is that of double Mach reflexion, typical of the pseudostationary experiment at high values of $M_{\infty}$ and in the vicinity of the transition angle. The discontinuity line which separates particles processed by the two oblique shocks from those that have passed through the Mach stem leaves the triple point at a steep angle towards the wedge face. It is weakly indicated by a black fringe. The reflected shock is virtually straight and almost parallel to the wedge face. Figure $7(c)$ also shows, on the bottom surface $\left(\alpha=81.5^{\circ}\right)$, the situation encountered when $\alpha$ is further from transition, where simple Mach reflexion is seen to occur.

The transition angle was measured from these and a number of other interferograms to be

$$
\alpha_{\mathrm{tr}}=\left\{\begin{array}{l}
50 \pm 1^{\circ} \text { at } p_{1}=10 \text { torr, } M_{s}=12 \\
51.5 \pm 1^{\circ} \text { at } p_{1}=5 \text { torr, } M_{s}=12.9
\end{array}\right.
$$



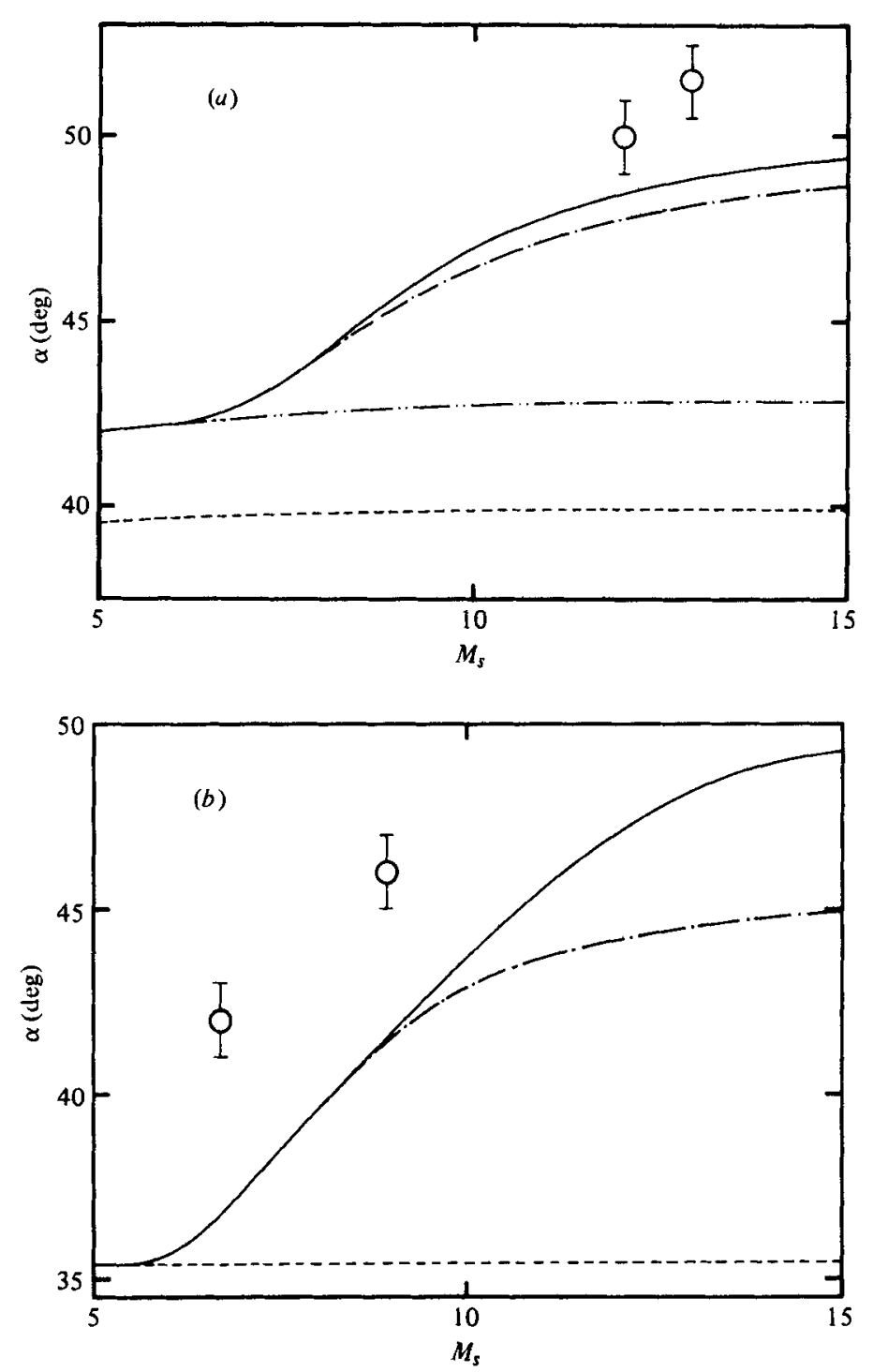

Figure 9. Comparison of measured transition angle (circles) with calculated sonic criterion $\left(\alpha_{\mathrm{s}}\right)$ for $(a)$ nitrogen $\left(p_{1}=10\right.$ and 5 torr) and $(b)$ argon $\left(p_{1}=10\right.$ torr) in pseudosteady flow. Calculated values:,$- I$ frozen, $R$ equilibrium; - - - $I$ equilibrium, $R$ equilibrium; $\cdots$..., perfect gas; - - . - $I$ frozen, $R$ frozen, vibration equilibrium.

Both values lie slightly but significantly above the predicted value of the sonic criterion for a frozen incident and equilibrium reflected shock (see figure $9 a$ ).

The interpretation of the fringe shift in these interferograms would be very difficult because it is complicated by the three-dimensional effects of an axisymmetrically bounded flow over a plane model of relatively small width. Also, the fringes visible behind the curved shock in figure $7(b)$, for example, are displaced from each other mainly because of the misalignment. Thus measurements of relaxation effects from these photographs are clearly not possible. However, by arranging a laser differential interferometer to measure the density as a function of time near the tip of the wedge, 
the three-dimensional effects may be considerably reduced. This technique is described in detail by Oertel (1976). The results of applying it here confirmed the calculated density profile after the incident shock within the accuracy of the technique in this situation $( \pm 5 \%)$.

Typical results obtained in argon at $M_{s}=6 \cdot 7$ and $p_{1}=10$ torr are presented in figure 8 (plate 2). These show the transition from regular to double Mach reflexion, with incipient transition (indicated by the curvature of the reflected shock near the reflexion point) in figure $8(a)$ at $\alpha=42^{\circ}$ and two stages of double Mach reflexion at $\alpha=44^{\circ}$ and $46^{\circ}$ in figures $8(b)$ and $(c)$. The latter clearly show the virtually straight reflected shock, almost parallel to the wall, and the steep discontinuity line terminating in a vortex at the wedge surface. These argon experiments give the failure of regular reflexion at $42^{\circ}$ while another set at $M_{s}=9$ and $p_{1}=10$ torr gives it at $46 \cdot 2^{\circ}$. At both these conditions, almost no ionization occurs after the incident shock, while the reflected shock produces a significant amount of ionization. The real-gas effect in this situation is therefore similar to that of a frozen incident and an equilibrium reflected shock in nitrogen and in fact the ionization relaxation length after the reflected shock is so small that the argon may be considered to be in equilibrium. The regular reflexions give reflected shock angles approximately $2^{\circ}$ higher than the calculated values. The calculated values of the sonic condition for equilibrium argon as well as that for argon as a perfect gas $\left(\gamma=\frac{5}{3}\right)$ are compared with experimental results in figure $9(b)$. Both experimental points lie approximately $5^{\circ}$ above the equilibrium sonic criterion. This excess is larger than in the case of nitrogen. In all cases it is thought to be due to the displacement effect of the boundary layer after the reflected shock, which will be discussed in \$4. A further contribution arises from the non-uniformity of conditions after the two shock waves caused by finite relaxation lengths, which are not accounted for in the calculations of $\alpha_{s}$. This non-uniform region is called the entropy layer (see Oertel 1974).

\section{The steady case}

\subsection{Facility, experimental conditions and technique}

In order to produce a steady flow at high Mach number the facility described in $\$ 2.2$ was used in its shock-tunnel mode. In this configuration the primary shock wave is reflected off the end of the shock tube, thus producing a high enthalpy reservoir which is then expanded through a contoured nozzle of exit diameter $25 \mathrm{~cm}$ into a test section and dump tank.

A number of constraints influenced the choice of experimental conditions. In order to obtain strongly evident real-gas effects, the reservoir enthalpy has to be comparable to the dissociation energy of the gas. However, as the enthalpy is increased, the dissociation rate rises rapidly, so that the relaxation length becomes small. Even more important is the limitation on the running time imposed by the earlier onset of drivergas contamination at higher enthalpy. A number of previous experiments in the facility (see Hornung 1972; Ebrahim \& Hornung 1975; Ebrahim \& Sandeman 1976; Crane \& Stalker 1977; Kewley \& Hornung 1974b) had shown that the conditions chosen for the present experiments (carbon dioxide at $3.6 \mathrm{~km} / \mathrm{s}, 3.8 \times 10^{-6} \mathrm{~g} \mathrm{~cm}^{-3}, M_{\infty}=5.5$, and nitrogen at $5 \cdot 1 \mathrm{~km} / \mathrm{s}, 2 \times 10^{-6} \mathrm{~g} \mathrm{~cm}^{-8}, M_{\infty}=7 \cdot 2$ ) are safe. 'Safe' in this context means that measured quantities such as density, temperature, composition and pressure 
agree with numerically computed values. At these conditions the incident shock in Mach-reflexion experiments produces only slight dissociation, so that only the relaxation after the reflected shock produces significant real-gas effects. The relaxation lengths after the reflected shocks are short, however, being less than $1 \mathrm{~cm}$ in both cases. Another feature of these conditions is that the contoured nozzle, designed for air as the test gas at a specific reservoir enthalpy of $3 \times 10^{7} \mathrm{~m}^{2} / \mathrm{s}^{2}$ and reservoir pressure of 200 atm, gives uniform flow conditions for both of the selected conditions in nitrogen and carbon dioxide. It should be pointed out that since the test gas is dissociated in the reservoir it recombines rapidly on cooling near and after the nozzle throat, until its composition is frozen by the rapid drop in density through the nozzle flow. This results in different ratios of specific heats for different gases, thus making it possible to obtain different exit Mach numbers for different gases. The same nozzle also gives reasonably good uniform conditions with argon as a perfect gas, producing an approximate exit Mach number of 16. This was exploited in our experiments in order to check the transition condition for a perfect gas.

The steady flow experiment has the favourable feature that the shock-reflecting wall, and therefore the effects of the boundary layer, may be eliminated by symmetry, two shock-generating wedges being arranged as mirror images of each other in a plane parallel to the free-stream direction so that this plane is tantamount to an inviscid wall (see figure 11, plate 3). The experiments of Hornung \& Kychakoff (1977) had shown that for accurate work it is essential to be able to adjust both the spacing and the inclination of the wedges continuously. Accordingly a model was constructed in which these adjustments are measured on vernier scales giving the inclinations to within $\pm 0.1^{\circ}$. The wedges have a width across the flow of $15 \mathrm{~cm}$ and a face length of $4 \cdot 3 \mathrm{~cm}$.

Mach-Zehnder interferometry again served to visualize the flow, the light source being an exploding wire focused on a $1.5 \mathrm{~mm}$ diameter aperture and triggered from the last shock timing station with an appropriate delay. The flash from the exploding wire lasts for approximately $50 \mu \mathrm{s}$, which is sufficiently short compared with the duration of uniform conditions in the run (approximately $500 \mu \mathrm{s}$ ). A suitable portion of the spectrum of this light (free of impurity lines) was selected by an interference filter passing wavelengths of $510 \pm 5 \mathrm{~nm}$, which was inserted in the beam near the image of the light source made by the output lens of the interferometer and just before the camera.

Since the damage suffered by the windows from diaphragm fragments is not so serious in this configuration of the facility, better quality windows could be used than in the pseudosteady experiments. Any small faults in the windows also do not show up as badly in the interferograms, because the exploding-wire light source does not have the transverse coherence present in the dye laser beam, which caused the diffraction patterns around window imperfections in the interferograms of figures 7 and 8 .

The steady flow experiments essentially repeat the results of Hornung \& Kychakoff (1977) with greater accuracy, and a more nearly two-dimensional configuration. In addition, however, the spacing of the trailing edges of the shock-generating wedges could be adjusted systematically in order to vary the distance behind the reflexion point at which the leading characteristic from the trailing edge strikes the centreline. The ratio of this distance to the relaxation length after the reflected shock was expected to affect the transition. 


\subsection{Results}

The first experiment with the new, more accurately adjustable model was to verify that transition occurs at the von Neumann condition for a perfect gas in steady flow. In argon flow at a specific reservoir enthalpy of $1.7 \times 10^{6} \mathrm{~m}^{2} \mathrm{~s}^{-2}$, reservoir pressure of $260 \mathrm{~atm}$ and a nominal Mach number of 16 , transition was observed at a wedge-angle setting of $18 \cdot 6 \pm 0 \cdot 3^{\circ}$. At this condition the boundary layer on the wedge is quite thick and may easily be seen in the interferogram of figure 10 (plate 3). However, the shock reflexion point in the symmetrical arrangement is remote from the boundary layer and the shocks are sufficiently straight at the reflexion point to get a good measurement of the angle. At transition $\alpha$ was measured to be $27 \cdot 0 \pm 0 \cdot 6^{\circ}$, in reasonable agreement with the von Neumann condition, $\alpha_{N}=26 \cdot 1^{\circ}$, and well below the detachment criterion, $\alpha_{d}=35 \cdot 5^{\circ}$.

The transition condition in the case of a dissociating nitrogen flow is shown in the two interferograms of figure 11 (plate 3 ). The difference in the wedge inclinations between the two interferograms is only $0 \cdot 2^{\circ}$, and there is no measurable difference in the incident shock angles. The large Mach-stem length produced by such a small change in wedge angle is in contrast with the behaviour observed by Hornung \& Kychakoff (1977). The main difference between the two experiments is the aspect ratio of the wedges, the streamwise length $w$ of the wedge face being shorter in the present experiments so that end effects are less important. In figure $11(b)$ the flow behind the incident shock shows evidence of a very slight amount of relaxation by the variation of blackness with distance from the shock. This is not visible in figure $11(a)$ because the interferometer setting is such that the post-shock density does not quite bring the condition to the most sensitive part of the intensity curve. The relaxation after the reflected shock causes a shift of approximately 2.5 fringes over a relaxation length of $0.7 \mathrm{~cm}$, after which the expansion from the trailing edge of the wedge causes the density to drop again.

Figure $11(b)$ clearly shows the initially converging streamlines through the two triple points, which can be seen to form a throat under the influence of the two expansion fans. The flow after the Mach stem therefore consists of an initially dominating subsonic relaxation with rising density and pressure, followed by a convergent subsonic and supersonic divergent flow with a density drop. The density maximum occurs in the wide white fringe parallel to the Mach stem.

The features of the nitrogen results are repeated in the three sets of interferograms of figure 12 (plate 4) for dissociating carbon dioxide flows, in which the wedge spacing has been varied to change the distance $c$ between the regular reflexion point and the point where the leading characteristic from the trailing edge of the wedge strikes the centre-line (see figure $13 a$ ). The relaxation length is slightly smaller than in the nitrogen flow and the fringe shift is considerably larger. The latter feature makes it much easier to see the slight relaxation effect after the first shock and the strong relaxation after the reflected shock and Mach stem. The streamlines through the triple points are again clearly evident. The incident shocks are virtually straight, the slight relaxation after them causing only minimal curvature, whereas the reflected shocks show fairly strong convex curvature very near the reflexion point which is more spread out for the less deflected flow of the Mach reflexions.

Again the Mach-stem length increases rapidly at transition. This may be seen more 

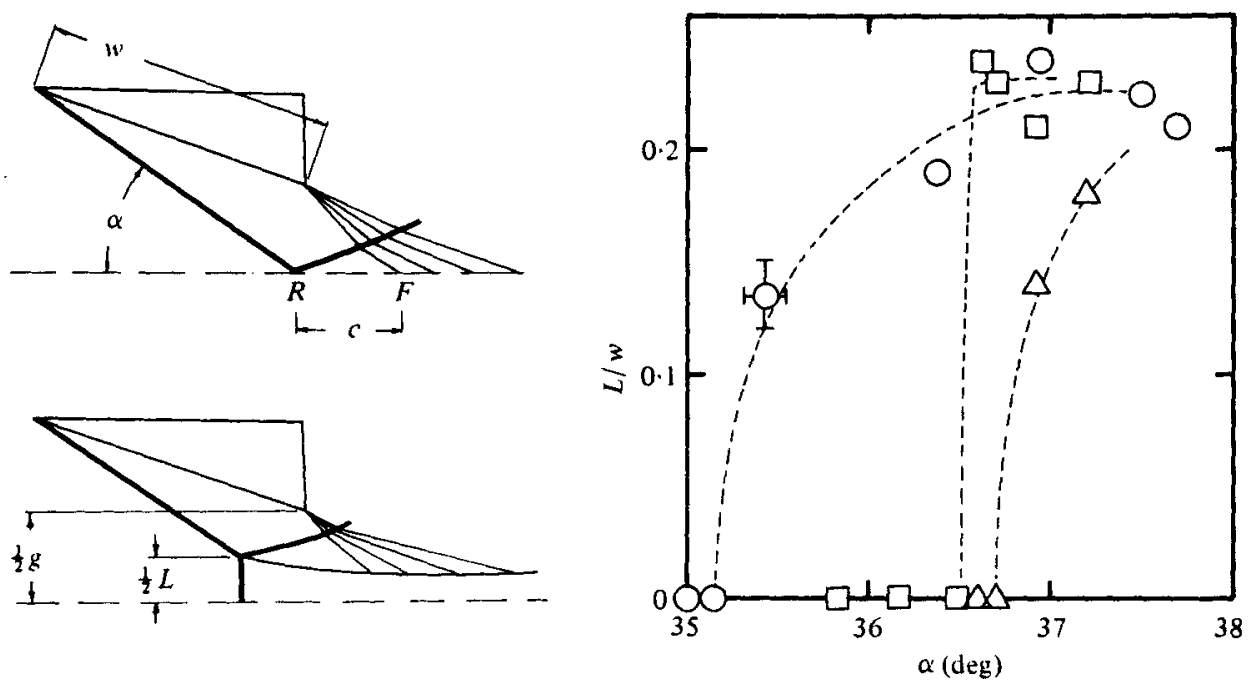

(d)

(b)

Fraure 13. Mach-stem length during the transition process in steady flows of dissociating carbon dioxide shown in figure 12. $O, g / w=0.56 ; \square, g / w=0.37 ; \Delta, g / w=0.84 ;-.$, mean lines through points. The sketches in $(a)$ define relevant dimensions.

clearly in the plot of the dimensionless Mach-stem length against $\alpha$ shown in figure 13 for three values of the gap $g$ between the trailing edges of the wedges. For the smallest gap, $c$ is largest and the flow after the regular reflexion has reached equilibrium before the leading characteristic of the expansion fan reaches the centre-line. A sharp transition is observed at $\alpha=36 \cdot 3^{\circ} \pm 0 \cdot 2^{\circ}$. As the gap is increased, the transition becomes less sharp and occurs at a smaller angle $\alpha=35 \cdot 2 \pm 0 \cdot 2^{\circ}$. However, for a still larger gap, the transition angle increases again. The expected monotonic reduction of the transition angle with decreasing $c$ does not seem to occur.

Similar results were also obtained in nitrogen flow, and a summary of all the result is given in table 1. This also gives the calculated equilibrium transition condition according to the von Neumann criterion. Although there is evidence of a dependence of $\alpha_{\mathrm{tr}}$ on $g / w$ there is reasonable agreement between the experimental result and the calculated von Neumann condition in all cases except that of carbon dioxide. However, the vibrational state of this gas in the free stream is quite important and somewhat uncertain, causing an uncertainty of $\pm 1^{\circ}$ in the calculated $\alpha_{N}$.

An interesting phenomenon can be observed in most regular-reflexion interferograms and is evident more clearly in the regular reflexion in carbon dioxide at

$$
g / w=0.37
$$

of figure 12. This is a very sharp minimum in a transverse density profile, on the centreline just after the reflexion point, evidenced by a sharp deflexion of the fringes in that region. Although it is possible that this is due to the relaxation effect described by Buggisch (1973), that particles passing through the two shocks further from the centre-line spend more time between the shocks and therefore eventually reach a different equilibrium state, the present results are unsuitable to test this conclusively. 


\begin{tabular}{|c|c|c|c|}
\hline Gas & $g / w$ & $\alpha_{\mathrm{tr}}$ (measured) & $\begin{array}{c}\alpha_{N} \\
\text { (calculated) }\end{array}$ \\
\hline Argon (perfect gas) & $0 \cdot 41$ & $27 \cdot 0 \pm 0 \cdot 6^{\circ}$ & $26 \cdot 1^{\circ}$ \\
\hline Nitrogen (dissociating) & $\begin{array}{l}0.27 \\
0.36 \\
0 \cdot 67\end{array}$ & $\left.\begin{array}{l}32 \cdot 1 \pm 0 \cdot 2^{\circ} \\
33 \cdot 1 \pm 0 \cdot 2^{\circ} \\
33 \cdot 3 \pm 0 \cdot 2^{\circ}\end{array}\right\}$ & $32 \cdot 6^{\circ}$ \\
\hline Carbon dioxide (dissociating) & $\begin{array}{l}0.37 \\
0.56 \\
0.84\end{array}$ & $\left.\begin{array}{l}36 \cdot 5 \pm 0 \cdot 2^{\circ} \\
35 \cdot 2 \pm 0 \cdot 2^{\circ} \\
36 \cdot 7 \pm 0 \cdot 2^{\circ}\end{array}\right\}$ & $35 \cdot 0^{\circ}$ \\
\hline
\end{tabular}

Table 1. Transition angle in steady flow.

\section{Discussion}

\subsection{The displacement effect in pseudosteady flow}

In the pseudosteady flow the condition at which a pressure signal from the wedge tip can just keep pace with the reflexion point occurs at $\alpha=\alpha_{g}$, at which the flow after the reflected shock travels at the sonic velocity relative to the reflexion point. Accordingly, the length scale of the boundary conditions may not be communicated to the reflexion point when $\alpha<\alpha_{s}$. Also, regular reflexion cannot exist above $\alpha=\alpha_{d}$. The angles $\alpha_{s}$ and $\alpha_{d}$ are separated only by a very small amount $\left(\sim 0 \cdot 1^{\circ}\right)$, so that from an experimental point of view they may be considered coincident. Yet the experimental results for both dissociating nitrogen and ionizing argon lie well above the calculated value of $\alpha_{d}$. It was suggested in $\$ 2$ that this discrepancy is due to the displacement effect of the boundary layer on the wedge and the entropy layer. This section gives a discussion of the reasons for this explanation.

The evidence of previous experimenters shows that $\alpha_{t r}$ is always observed to exceed $\alpha_{d}$ in reflexion off a wedge. Bleakney \& Taub (1949), for example, measured an approximately constant value of $\alpha_{\mathrm{tr}}-\alpha_{d}$ of $2^{\circ}$ in the range $1<M_{s}<3$. In a recent investigation of shock reflexion from a cone, Takayama \& Sekiguchi (1977) observed values for $\alpha_{\mathrm{tr}}-\alpha_{d}$ which increased uniformly with $M_{s}$ up to $6^{\circ}$ at $M_{s}=1.53$ and then dropped back to $3^{\circ}$ at $M_{s}=1.8$ and 3.1. It turns out that the shock-tube driver condition was altered (to a higher pressure) at $M_{s}=1 \cdot 8$. At a constant driver condition, reduction of the Reynolds number is consistent with an increase in Mach number, and the change in the driver condition would increase the Reynolds number again. The best way to test the idea that viscosity is responsible for the excess, however, is to eliminate the wall. This was done by Henderson \& Lozzi (1975), who placed two wedges symmetrically in the path of a plane shock so that the two reflected shocks collided. The interaction is identical to the reflexion off a wedge without the no-slip condition at the wall. Henderson \& Lozzi $(1975$, figures $5 a, b)$ observed that with a wall $\alpha_{\mathrm{tr}}-\alpha_{d}=2^{\circ}$ and that without a wall $\alpha_{\mathrm{tr}}-\alpha_{d}=-0.5^{\circ}$. The error bar on their angle measurements is $\pm 0.5^{\circ}$. The removal of the displacement effect therefore removes the discrepancy. The wall-less pseudostationary experiment was also performed very carefully by Smith (1959) at low Mach numbers, and excellent agreement with almost identical sonic and detachment criteria was observed. None of these results caused their discoverers to associate the excess with transport properties, however, and they did not even quote the Reynolds number of their experiments. 


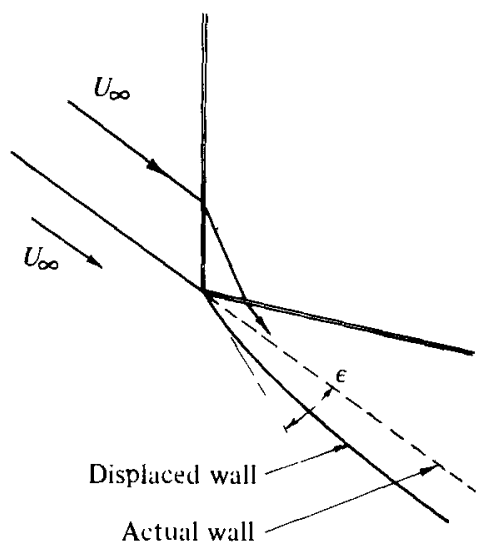

(a)

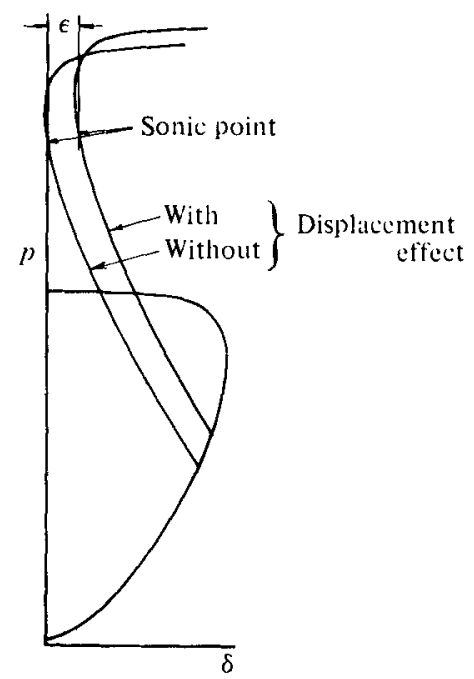

(b)

Figure 14. The displacement effect. (a) Concave displaced wall due to the negative displacement thickness of the boundary layer. (b) Effect on the $p, \delta$ map.

The mechanism by which the viscosity and heat conduction modify the transition condition may be explained by considering the regular reflexion in the frame of reference of the reflexion point. A uniform flow strikes the incident shock at an angle $\alpha$ and parallel to the wall, whose velocity $U_{\infty}$ is the same as that of the uniform flow (see figure 14): After the reflected shock, the flow velocity is lower than $U_{\infty}$ but the wall velocity remains at $U_{\infty}$. The region affected by this shear (i.e. the boundary layer) grows owing to the action of viscosity, and since the wall moves faster than the flow, the displacement thickness is negative. The deflexion through the reflected shock necessary to bring the flow into the direction of the 'displaced wall' is therefore less than that required with inviscid flow. How this affects the transition condition may be demonstrated in the $p, \delta$ plane (figure 14b). The reflected shock does not now have to return the flow to $\delta=0$ but only to some finite value $\epsilon$ characteristic of the growth rate of the displacement thickness at a point given by the limit of resolution of the experimenter.

Clearly this raises the value of $\alpha_{\mathrm{tr}}$ by an amount which, to a first approximation, may be expected to be proportional to $\epsilon$. Using the results of Knöös (1968), the rate of growth of displacement thickness in our experiment may be estimated: at $1 \mathrm{~mm}$ from the reflexion point, $\epsilon=1^{\circ}$ and $1 \cdot 6^{\circ}$, respectively, for the two nitrogen experiments; $\epsilon=2 \cdot 6^{\circ}$ for both the argon experiments. These estimates can be seen to follow the variation of the measured excess with the conditions quite closely $\left(\alpha_{\operatorname{tr}}-\alpha_{s}=1.6^{\circ}\right.$, $2 \cdot 7^{\circ}$ and $5^{\circ}$ respectively).

In response to this effect the angle made by the reflected shock with the wall should decrease. The opposite is in fact observed: the detailed measurements by Oertel (1974) in oxygen, the regular reflexion in argon as well as the present nitrogen results all give a consistently high reflected shock angle though the discrepancy is barely significant in the last case. A satisfactory explanation has yet to be found.

At this point it is appropriate to remember that in some experiments the excess of 
$\alpha_{\mathrm{tr}}$ over $\alpha_{d}$ is so large that it can hardly be explained in terms of viscous effects. Thus Heilig (1969) measured $\alpha_{\mathrm{tr}}-\alpha_{d}$ up to $13^{\circ}$ in experiments on reflexion from spheres and circular cylinders. However, since these are properly unsteady experiments, the sonic speed and the flow speed after the reflected shock both vary with time, and the propagation of information about the first contact of the incident shock with the body lags further behind the reflexion point even on inviscid grounds. This view is consistent with the results of experiments by Henderson \& Lozzi (1975, figure 7) on shock reflexion from convex and concave models, the former giving a large excess and the latter yielding transition at $\alpha_{N}$ !

Returning to figure 14(a), we observe that the concave shape of the displaced wall after the reflexion point may be the reason for the lack of curvature of the regularly reflected shock observed by Oertel (1976) in flows with finite relaxation length, after the reflected shock. The displaced wall shape may also be responsible for the occurrence of double Mach reflexion under some circumstances.

\subsection{The transition criterion}

The results for steady flow transition to Mach reflexion of Henderson \& Lozzi (1975, figure 4) and of Hornung \& Kychakoff (1977) as well as the present results confirm the von Neumann criterion unambiguously. Conditions at this lower value of $\alpha$ must therefore be such that, in steady flow, the length scale of the boundary conditions is able to communicate itself to the reflexion point. As the flow is everywhere supersonic at $\alpha<\alpha_{s}$, however, this does not seem possible.

It is more profitable to consider the relative stability of Mach reflexion and regular reflexion when $\alpha_{N}<\alpha<\alpha_{d}$ in steady flow. If, through some disturbance, for example during the tunnel starting process, a Mach reflexion is temporarily established, the flow after the Mach stem is subsonic until it is accelerated again by the expansion fan from the trailing edge of the wedge (see, for example, figure 11b). A path communicating the length scale of the boundary conditions (i.e. the wedge length) to the reflexion point therefore exists, and Mach reflexion is stable. Mach reflexion cannot exist, however, below $\alpha=\alpha_{N}$ (see, for example, Courant \& Friedrichs 1948). Hence, with a history of sufficiently large disturbances, say during a suitable starting process, $\alpha_{\mathrm{tr}}=\alpha_{N}$ for stationary flow.

In the pseudosteady case it is not certain whether the same stability argument applies. If, through some disturbance, a small Mach reflexion is set up, the subsonic region behind it is limited in extent, and it may be that a communication path is not set up by it whereby the length scale can be transmitted to the reflexion point. The Mach reflexion may therefore be unstable in $\alpha_{N}<\alpha<\alpha_{d}$. However, it may be that the regular reflexion merely persists because a suitable disturbance is not available in pseudosteady flow. Another way of viewing this is to observe that the length scale of the boundary conditions now increases linearly with time. The signal from the leading edge of the wedge can keep pace with the reflexion point only if the velocity relative to the reflexion point is sonic or smaller. Hence $\alpha_{\mathrm{tr}}=\alpha_{s}$ for pseudosteady flow.

The evidence and the above explanation may be summarized by a single transition criterion: in steady or pseudosteady flow, transition to Mach reflexion occurs at that angle $\alpha_{\mathrm{tr}} \geqslant \alpha_{N}$ at which conditions change in such a way as to open an information path enabling the communication of a length scale to the reflexion point. 


\subsection{The effect of relaxation}

Both the pseudosteady and the steady flow experiments yielded results which indicate that the equilibrium (incident shock frozen) transition conditions apply. They were therefore not able to resolve effects on the transition angle due to finite relaxation length, so that part of the following discussion is somewhat speculative.

The steady flow experiments set out to investigate the effect of relaxation by altering the distance $c$; see figure $13(a)$. As this distance is decreased, a condition is reached at which the point $F$ lies within the relaxation region after the reflected shock, and an effect on the transition may be expected. Unfortunately, the relaxation distance was so small in our experiments that a large gap $g$ was necessary to bring the point $F$ close enough to $R$. This meant that the expansion was much more diffuse for small $c$ than for large $c$. Consequently the position of the throat formed by the two slipstreamlines was not altered significantly by changing $g$. Since the distance to this throat is likely to be the important length scale, the experiment should ideally be performed by reducing the scale of the boundary conditions while maintaining geometric similarity and constant relaxation length. (This would certainly have to yield a different transition condition, namely $\alpha_{t r}=\alpha_{N, \text { fr }}$, in the limit when $w$ becomes much smaller than the relaxation length.) The experiments do show a barely significant variation of $\alpha_{\mathrm{tr}}$ with $g / w$ (see figure 13(b) and table 1), which does not follow the expectation that $\alpha_{t r}$ should decrease as $g / w$ is increased at constant relaxation length.

If the relaxation length is smaller than $c$ and if the inequalities (2) apply, an interesting effect of relaxation may be expected in steady flow: as $\alpha$ is increased, transition to a small Mach reflexion should occur at $\alpha=\alpha_{d, \text { fr }}$, the subsonic flow after the Mach stem being accelerated to supersonic conditions again by the pressure drop fed down from the curved reflected shock. The Mach-stem length will be governed by the distance to the sonic point, i.e., by the relaxation length. As $\alpha$ is increased a point will be reached where the relaxation can no longer accelerate the flow to supersonic conditions, and $w$ will become the governing length scale.

In the pseudosteady case the influence on $\alpha_{s}$ of relaxation between the two shock waves is not very important, as may be seen from figure 6 . However, an interesting effect may be expected when the relaxation length after the reflected shock is finite: as $\alpha$ is increased through $\alpha_{d, \mathrm{rr}}$ (inviscid flow) the flow immediately behind the reflected shock becomes subsonic relative to the reflexion point and is accelerated again to supersonic by the relaxation. The distance to the sonic point constitutes a new length scale governed by the relaxation length. Thus a Mach reflexion will be generated with $L$ proportional to the distance from the reflexion point to the sonic point. As $\alpha$ is increased, the distance to the sonic point increases, and therefore $L$ increases, until $\alpha_{d, \text { eq }}$ is reached, when the distance to the wedge tip becomes the governing length scale. (Parallel behaviour has recently been observed in the steady flow detachment process of the bow shock from a wedge in the presence of relaxation by Hornung \& Smith 1979.) This behaviour actually corresponds to that expected according to the argument of Hornung \& Kychakoff (1977), which, indeed, should be valid here since only one transition condition $\left(\alpha_{t r}=\alpha_{d}\right)$ is involved. 


\subsection{Interesting further experiments}

The need for a disturbance in the mechanism of transition to Mach reflexion in steady flow suggests the following experiment. If, in a steady flow facility (continuous supersonic wind tunnel) $\alpha$ is adjusted during the flow, the unstable situation of regular reflexion at $\alpha>\alpha_{N}$ should be maintainable as $\alpha$ is increased smoothly from $\alpha<\alpha_{N}$ until $\alpha=\alpha_{s}$. On the other hand, as $\alpha$ is decreased from $\alpha>\alpha_{d}$, Mach reflexion should continue to occur until $\alpha \leqslant \alpha_{N}$. This experiment would test the new criterion unambiguously.

To investigate the influence of relaxation, the steady flow experiments should be repeated by maintaining geometric similarity of the models while reducing their size, with a constant relaxation length larger than in the present experiments. In the pseudosteady case the situation when the relaxation length is finite after the reflected shock should be re-examined in more detail to determine if the behaviour described in $\$ 4.2$ occurs. Another relaxation effect could be studied by time-resolved interferometry of the pseudosteady flow around the time when the distance between the tip of the wedge and the reflexion point is comparable with the relaxation length.

The viscous displacement effect in pseudosteady flow could be studied by varying the Reynolds number. Argon is a particularly attractive gas for this purpose, since the effect is quite strong at easily achievable conditions. Also, the viscosity of argon has a maximum at approximately $12000^{\circ} \mathrm{K}$ and falls rapidly at higher temperatures, so that a high Reynolds number may be achieved at high $M_{s}$.

\section{Conclusions}

Transition from regular to Mach reflexion of shock waves was observed to occur at the von Neumann condition in steady flow and somewhat above (on the Mach-reflexion side of) the detachment condition in pseudosteady flow. The excess of the transition angle over the detachment angle is consistent with a negative displacement effect of the wall layer after the reflected shock in the present and in previous experiments though this is not supported by measured reflected shock angles. A single criterion explaining both sets of data as well as those of previous experimenters is that transition occurs when conditions change in such a way as to open an information path enabling the communication of a length scale to the reflexion point. On this basis a hysteresis effect is predicted in transition when the wedge angle is changed during a steady flow.

The effect of nitrogen dissociation with a finite length scale between the shocks and equilibrium after the reflected shock was examined in the pseudosteady case. The measured density profile after the incident shock confirmed calculations. Although calculations predict a small curvature in the regularly reflected shock, none could be detected. These and similar experiments with ionizing argon support the calculated detachment criterion, which lies up to $9^{\circ}$ above the perfect-gas value.

The effect of dissociative relaxation of nitrogen and carbon dioxide after the reflected shock was examined in symmetrical steady flow without a wall. The important length scale (the distance to the sonic point after the Mach stem) could not be made sufficiently small in relation to the relaxation length to obtain transition at anything but the equilibrium von Neumann condition. The experiments have given improved insight into the effect of relaxation however, enabling more useful experiments to be designed, some of which are suggested in $§ 4$. 
The project was given financial support by the Australian Research Grants Committee and by Deutsche Forschungs Gemeinschaft, which is gratefully acknowledged. The work also benefited from discussions with Professor E. Becker during his visit to ANU. Thanks are due to R. French, V. Adams and K. Smith for their technical support.

\section{REFERENCES}

Bleakney, W. \& Taub, A. H. 1949 Rev. Mod. Phys, 21, 584-605.

BUggisch, H. $1973 \mathrm{~J}$. Fluid Mech. 61, 159 172.

Courant, R. \& Friedrichs, K. O. 1948 Supersonic Flov and Shock Waves. Interscience.

Crane, K. C. A. \& Stalker, R. J: 1977 J. Phys. D, Appl. Phys. 10, 679.

Ebrahim. N. A. \& Hornung, H. G. 1975 A.I.A.A. J. 13, 845-846.

Ebratim, N. A. \& Sandeman, R. J. 1976 J. Chem. Phys. 65, 3446-3453.

Gyozdeva, L. G., Bazhenova, T. V., Predvoditeleva, O. A. \& Fokeev, F. P. 1969 Astron. Acta 14, 503-508.

Heilig, W. 1969 Dissertation, University of Karlsruhe. (See also Proc. 11th Int. Symp. Shock Tubes i Wares, Seattle, 1977, pp. 288-295.)

Henderson, L. F. \& Lozzi, A. 1975 J. Fluid Mech. 68, 139-155.

Horvung, H. G. 1972 J. Fluid Mech. 53, 149-176.

Hornung, H. G. \& Kychakoff, G. 1977 Proc. 11 th Int. Symp. Shock Tubes \& Waves, Seattle, pp. 296-302.

Hornong, H. G. \& Smith, G. 1979 J. Fluid Mech. To appear.

Kewley, D. J. \& Hornung, H. G. 1974 a Chem. Phys. Lett. 25, 531-536:

KewLEY, D. J. \& HoRnung, H. G. $1974 b$ J. Fluid Mech. 64, 725-736.

KNöös, S. 1968 J. Plasma Phys. 2, 207-242.

Landau, I. D. \& Lifshitz, E. M. 1959 Fluid Mechanics. Pergamon.

LAW, C. K. \& Glass, I. I. 1971 C.A.S.I. Trans, 4, 2.

Liepmand, H. W. \& Roshko, A. 1957 Elements of Gasdymamics. Wiley.

MACH, E. 1878 Akad. Wiss. Wien 77, 1228.

Neumann, J. von 1943 See Collected Works, 1963, vol. 6, pp. 239-299. Pergamon.

Oertet, H. 1974 Dissertation, Karlsruhe.

OERtel, H. $1976 J$. Fluid Mech. 74, 477-495.

Sмith, W. R. 1959 Phys. Fluids 2, 533-541.

STALKER, R. J. 1967 A.I.A.A.J. 5, 2160.

Takayama, K. \& Sekiguchi, H. 1977 Rep. Inst. High Speed Mech. Tohoku Univ. 336, 53-74. 

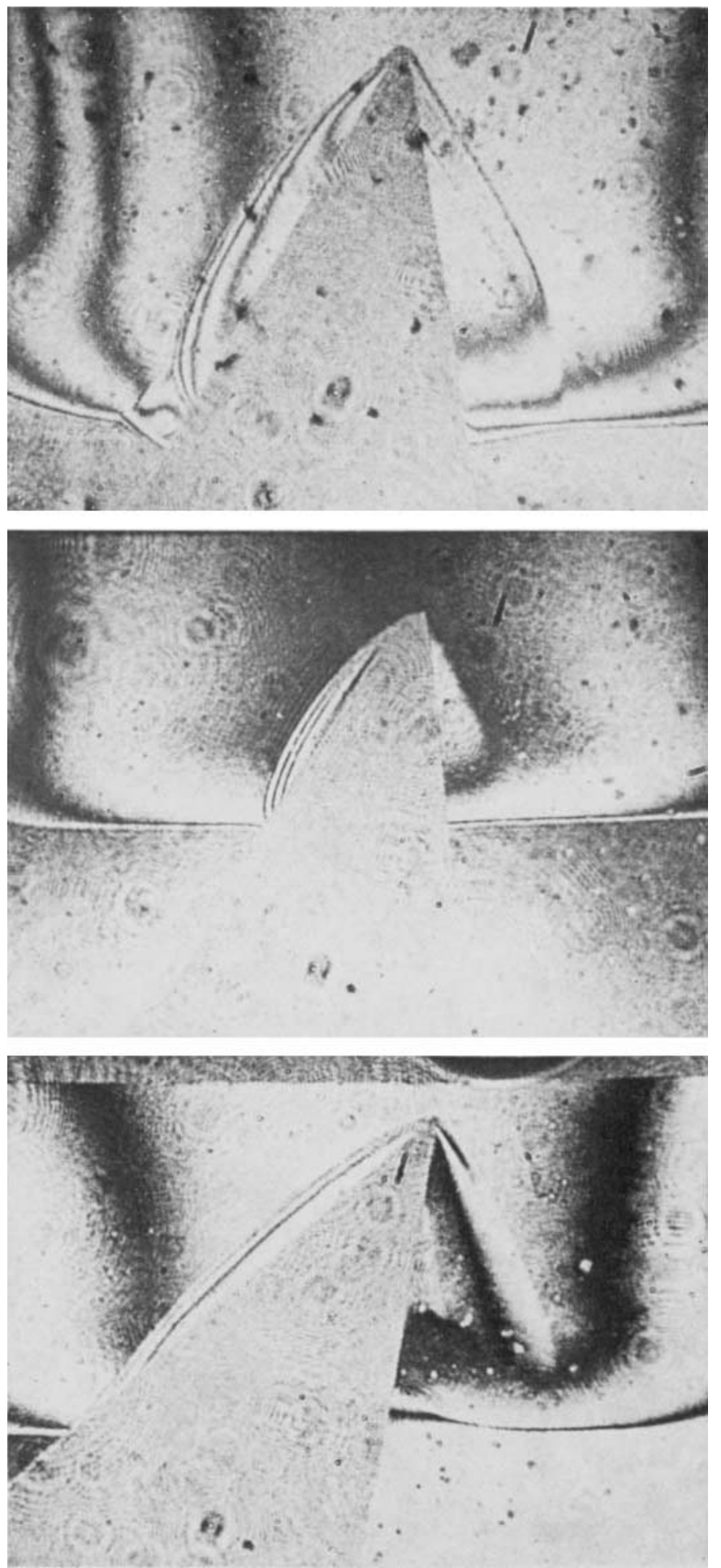

을

งิ

11.8

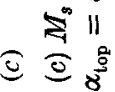

苟可

is

$11 . \frac{9}{x}$

र.

$\stackrel{\oplus}{\rightarrow}$

11

द

a

实

당

究

票

पू

3

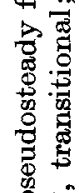
类

. ริ के 政 둥 छ

要。

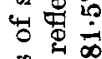

हैं

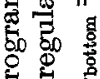

(3)

है II

$\therefore 8 \%$

오요

过

HORNUNG, OERTEL AND SANDEMAN 


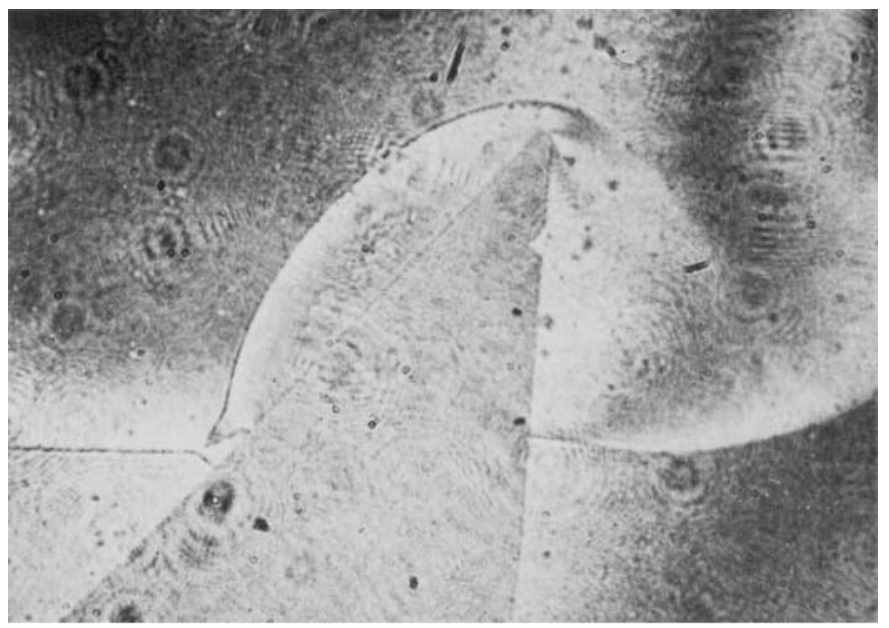

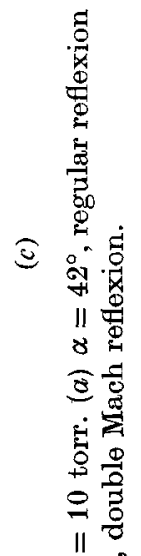
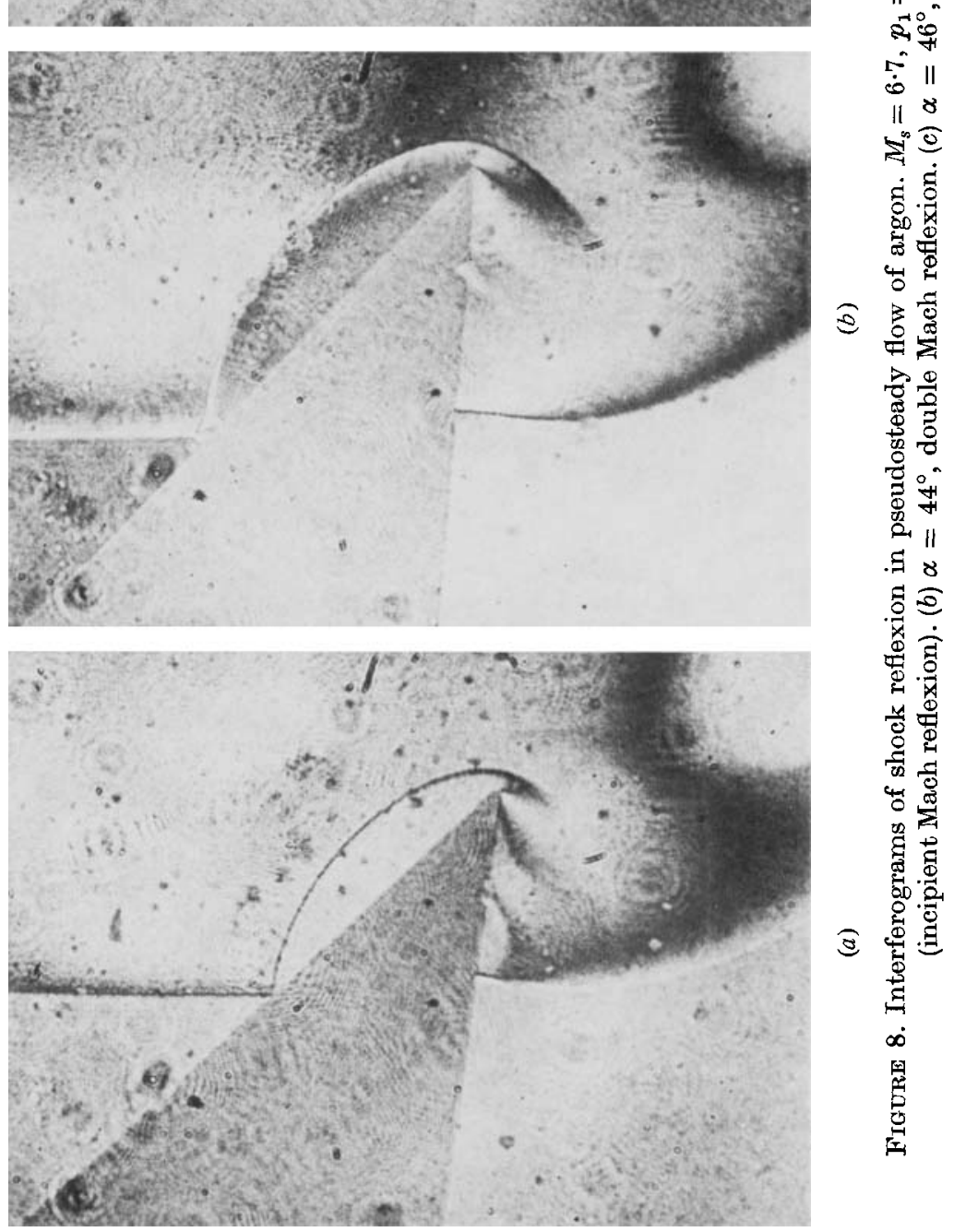

응

产

용

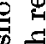

車

है

.

:

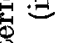

星

HORNUNG, OERTEL AND SANDEMAN 


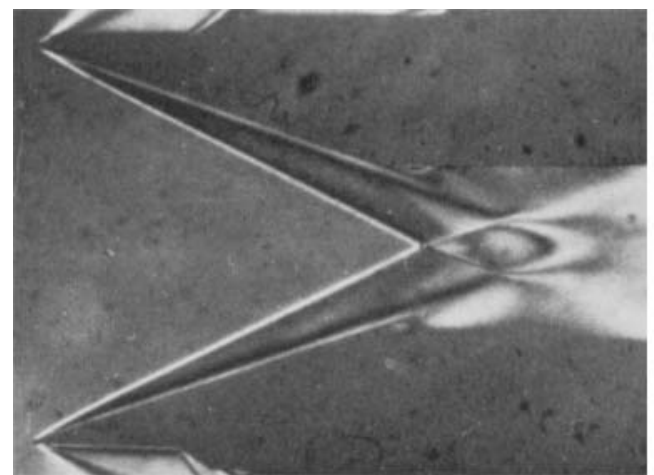

(a)

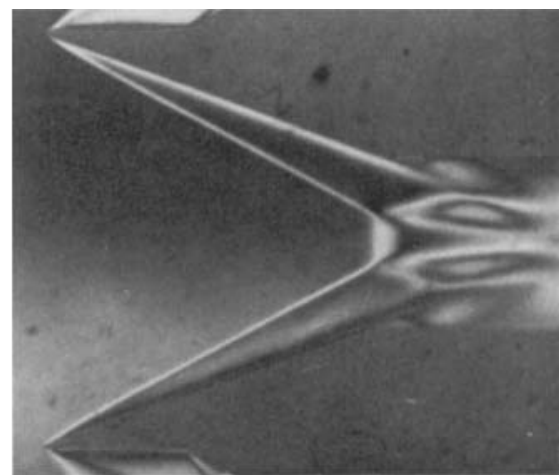

(b)

FigURE 10. Interferograms of steady flow shock reflexion. Perfect-gas argon, $M_{\infty}=16, g / w=$ $0 \cdot 41$. Note the thick boundary layer on the wedge surface. (a) Regular reflexion, $\alpha=26 \cdot 4^{\circ}$. (b) Mach reflexion, $\alpha=27 \cdot 6^{\circ}$.

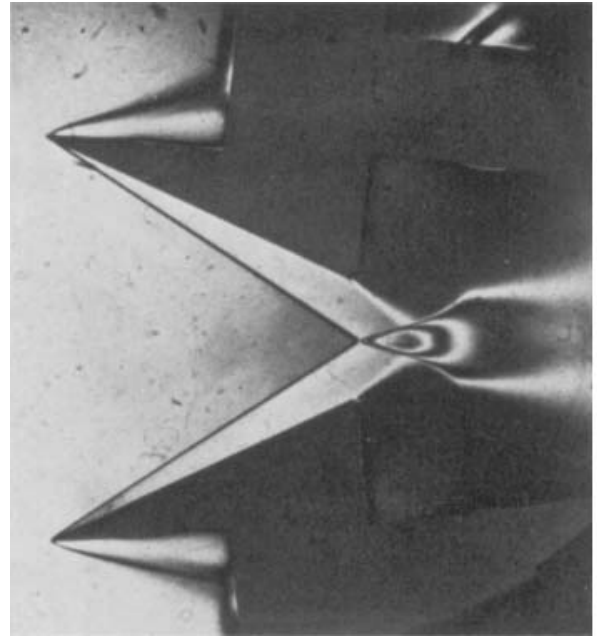

(a)

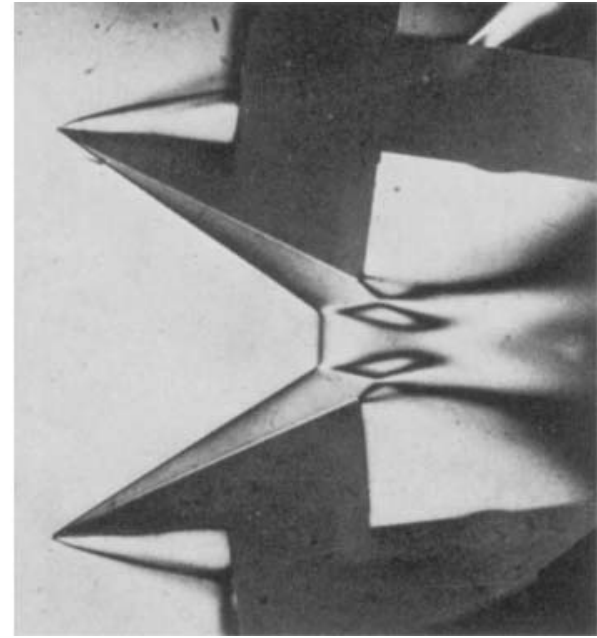

(b)

FIGURE 11. Interferograms of steady flow shock reflexion in dissociating nitrogen flow. $U_{\infty}=5 \cdot 1$ $\mathrm{km} \mathrm{s} \mathrm{s}^{-1}, \rho_{\infty}=2 \times 10^{-6} \mathrm{~g} \mathrm{~cm}^{-3}, M_{\infty}=7 \cdot 2, \mathrm{~g} / w=0.36$. Free-stream composition: $N_{2}, 0.0331$ mole/g; N, 0.00525 mole/g. (a) Regular reflexion, $\alpha=33 \cdot 1^{\circ}$; (b) Mach reflexion, $\alpha=33 \cdot 2^{\circ}$. 
Journal of Fluid Mechanics, Vol. 90, part 3

Plate 4

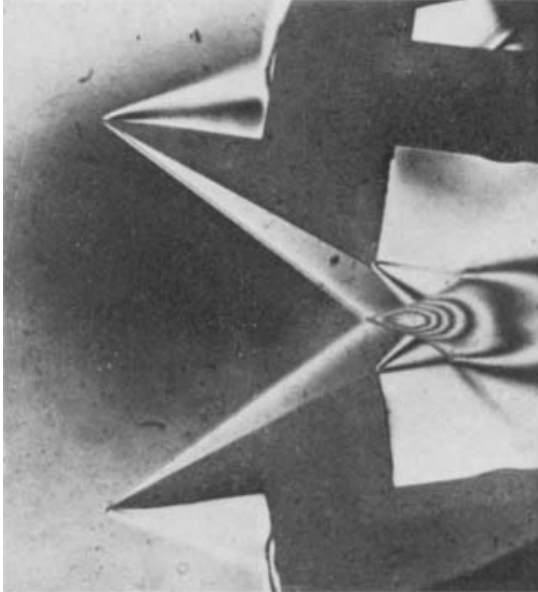

(a) $\alpha=36.2^{\circ}, g / w=0.37$

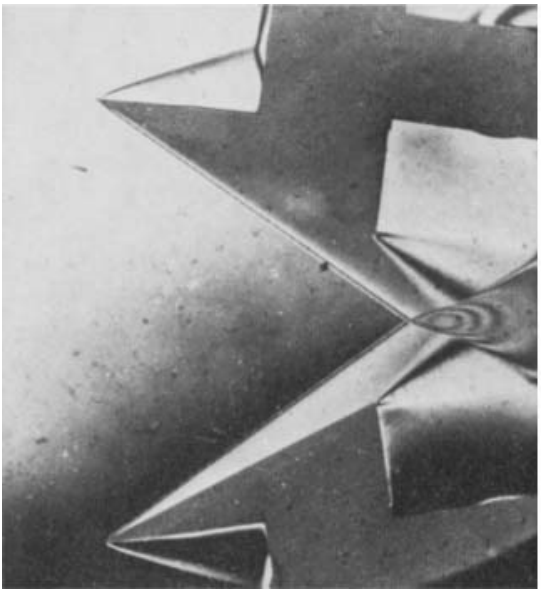

(c) $\alpha=35.1^{\circ}, g / w=0.56$

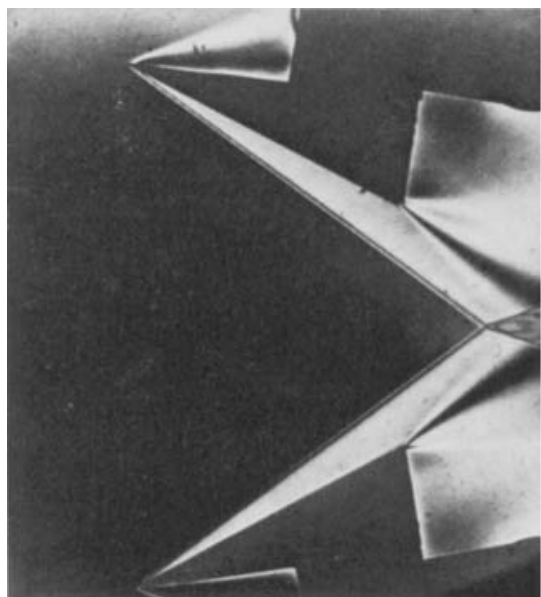

(e) $\alpha=36.6^{\circ}, g / w=0.83$

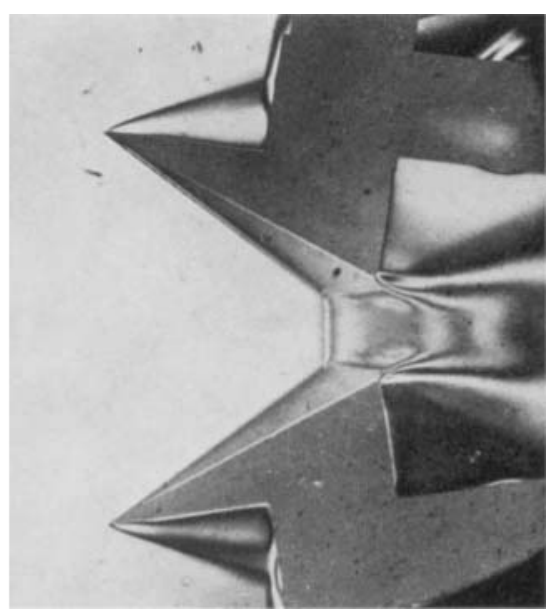

(b) $\alpha=36.5^{\circ}, g / w=0.37$

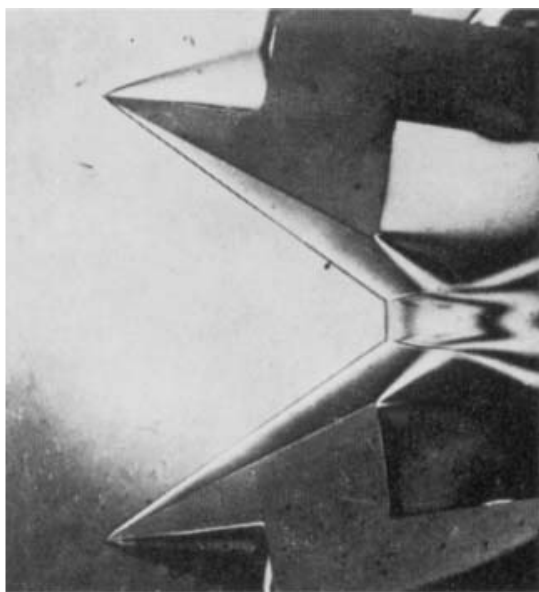

(d) $\alpha=35.3^{\circ}, g / w=0.56$

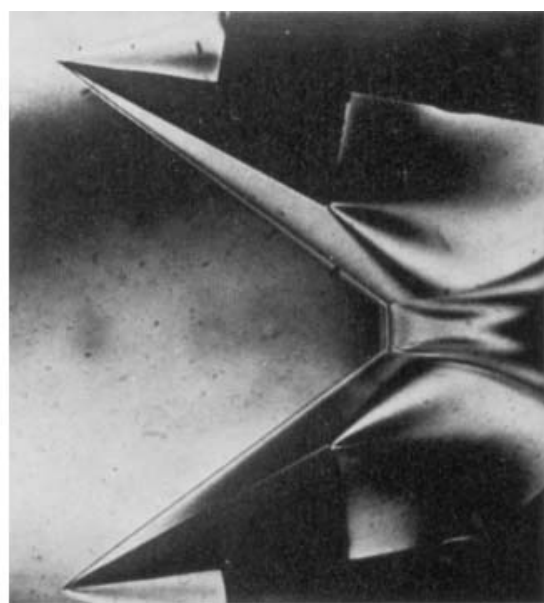

(f) $\alpha=36.8^{\circ}, g / w=0.83$

Frgure 12. Interferograms of steady-flow shock reflexion in dissociating carbon dioxide $U_{\infty}=3.6 \mathrm{~km} \mathrm{~s}^{-1}, \rho_{\infty}=3.8 \times 10^{-6} \mathrm{~g} \mathrm{~cm}^{-3}, M_{\infty}=5.5$. Free-stream composition: C, $10^{-11} \mathrm{~mole} / \mathrm{g} ; \mathrm{O}$, $6 \times 10^{-6} \mathrm{~mole} / \mathrm{g} ; \mathrm{CO}_{2}, 0.0089 \mathrm{~mole} / \mathrm{g} ; \mathrm{CO}, 0.0138 \mathrm{~mole} / \mathrm{g} ; \mathrm{O}_{2}, 0.0069 \mathrm{~mole} / \mathrm{g}$.

HORNUNG, OERTEL AND SANDEMAN 